\title{
Staff Attendance Monitoring System using Fingerprint Biometrics
}

\author{
M. Olagunju \\ Department of Computer Science, \\ Federal University Oye-Ekiti, Oye- \\ Ekiti, NIGERIA
}

\author{
A. E. Adeniyi \\ Department of Computer Science, \\ Landmark University, Omu-Aran \\ NIGERIA
}

\author{
T. O. Oladele \\ Department of Computer Science, \\ University of llorin, llorin, \\ NIGERIA
}

\begin{abstract}
Staff Attendance System is a simple windows based attendance system that was specifically developed for small and medium scale companies. This software helps to manage the workforce and track employee time and attendance in an easier way. This software application can manage the recordings, control and monitoring of staff absence and lateness. The significance of this application is to make sure that the staff member are punctual and do their jobs on time. Currently, there is no proper system to monitor the staff attendance at some companies. Some companies still use the paper based system to store the records of the employees. With the implementation of this system, paper based system will be eliminated. This research will help the Administrator to manage recordings, monitoring and tracking the attendance of the employees. It provides an accurate time management for the employees in order to sign in and sign out their attendance. In this paper, the biometrics based Staff Attendance Monitoring System was developed using Visual Basic Programming Language as front end while Microsoft Access was used as the Database to the backend users.
\end{abstract}

\section{General Terms}

Security, Fingerprint recognition, Attendance management

\section{Keywords}

Attendance, Staff, Monitoring System, Fingerprint, Biometrics

\section{INTRODUCTION}

"Time" they say is "Money". There is perhaps no more accurate analogy in business. Unfortunately, if there is one thing we all have difficulty managing, it is time. This includes the ability to accurately measure and manage the time and attendance of staff [1]. Indeed, new research from Aberdeen group reveals that time and attendance monitoring systems are now the very cornerstone for laying a foundation of effective workforce management systems. No wonder, then, that growing number of companies are eager in upgrading and adopting time and attendance systems.

Clients and colleagues count on staff to be at work as scheduled to provide smooth and efficient operations [7]. Although there are separate regulations governing different categories of staff, regular and punctual attendance is an ongoing expectation for all staff, both classified and professional The definition of the actual arrival time that constitutes a tardy or late occurrence is at the discretion of the supervisor, with consideration given to the type of position (for example, receptionist), client needs, and other relevant information. Failure to maintain an acceptable attendance record may result in an unsatisfactory performance evaluation and/or disciplinary action up to and including dismissal[10].
After visiting some organizations, it was discovered that there is no proper system to monitor the Staff attendance. Some companies still use log book to keep track of the employees' attendance. These attendance records are not precise. Besides, the company still uses the paper-based system to keep track of the records of the staff. This method is not secure because the records may be lost. It is also hard to find certain records using paper-based system. With the paper based system, the employee can also manipulate the time of signing in and out. The paper based system also waste a lot of time in signing in and out. The most important thing in having the system is to maintain an accurate and precise time management to track the attendance of the employees.

The developed system can trace the attendance of the employees so as to know when they are coming and leaving from work. Besides, the system is able to calculate the total working days of the employees, total of the employees who work over time, total of the employees who are coming late and also able to generate reports regarding the attendance of the employees.

With the report, the Administrator can easily analyze and detect the performance of the employees in the company. Besides, the system can keep track of the records about the employees such as their personal details. The administrator can view those records easily through this system without having to look into files manually. With the implementation of this system, the paper-based system will be eliminated. This system can save time and minimize the manpower for manual management. Besides, the employees' records are more secured which are saved into the database. This system also helps to reduce clerical cost such as papers, files and stationery.

Fingerprint may be deposited in natural secretions from the endocrine glands present in friction ridge skin (secretions consisting primarily of water) or they may be made by ink or other contaminants transferred from the peaks of friction skin ridges to a relatively smooth surface such as a fingerprint card [5]. The term fingerprint normally refers to impressions transferred from the pad on the last joint of fingers and thumbs, through fingerprint cards also typically record portions of lower joint areas of the fingers. An individual's fingerprint is scanned to identify 10 to 26 unique points of the finger, and a unique number is assigned to it.

Currently, there is no proper system to monitor the employees' attendance at some companies. Besides, the company still uses the paper-based system to keep track of the records of the employees. As a solution, the Fingerprint Biometrics Staff Attendance Monitoring system will be developed to overcome the problems that are stated above and provide an effective way to monitor the employees' attendance. This system will 
provide a user-friendly interface to make the system easy to use.

\section{LITERATURE REVIEW \\ 2.1 Biometrics}

Biometrics refers to the automatic identification of a person based on his physiological/behavioural characteristics [2]. This method of identification is preferred for various reasons; the person to be identified is required to be physically present at the point of identification. Identification based on biometric techniques obviates the need to remember a password or carry a token [13]. With the increased use of computers or vehicles of information technology, it is necessary to restrict access to sensitive or personal data. By replacing Personal Identification Number, biometric techniques can potentially prevent unauthorized access to fraudulent use of Automated Teller Machine, cellular phones, smart cards, desktop PCs, workstations, and computer networks. Personal Identification Number and passwords may be forgotten, and token based methods of identification like passports and driver's licenses may be forged, stolen, or lost. Thus, biometric systems of identification are enjoying a renewed interest. Various types of biometric systems are being used for real-time identification; the most popular are based on face recognition and fingerprint matching. However, there are other biometric systems that utilize iris and retinal scan, speech, facial thermo grams, and hand geometry.

A biometric system is essentially a pattern recognition system, which makes a personal identification by determining the authenticity of a specific physiological or behavioural characteristics possessed by the user [14]. An important issue in designing a practical system is to determine how an individual is identified. Depending on the context, a biometric system can be either a verification (authentication) system or an identification system. There are two different ways to resolve a person's identity: Verification and Identification. Verification (Am I whom I claim I am?) involves confirming or denying a person's claimed identity. In Identification one has to establish a person's identity (who am I?). Each one of these approaches has its own complexities and could probably be solved best by a certain biometric system.

\subsection{Fingerprint Scanner}

Perhaps most of the work in biometric identification has gone into the fingerprint. For general security and computer access control application fingerprints are gaining popularity [5]. The fingerprint's stability and uniqueness is well established. Based upon a century of examination, it is estimated that the change of two people, including twins, having the same print is less than one a billion. In verifying a print, many devices on the market analyze the position of details called minutiae such as the endpoi\{nts and junctions of print ridges. These devices assign locations to the minutiae using $\mathrm{x}, \mathrm{y}$, and directional variables. Some devices also count the number of ridges between minutiae to form the reference template. Several companies claim to be developing templates of fewer than 100 bytes. Other machines approach the finger as an image processing problem and applying custom very large scale integrated chips, neural networks, fuzzy logic and other technologies to the matching problem [15].

The fingerprint recognition technology was developed for some 12 years before being matched in 1983 by Identix Inc.
The Identix system uses a compact terminal that incorporates light and Charged Couple Device (CCD) image sensors to take high-resolution picture of a fingerprint. It is based on $68000 \mathrm{CPU}$ with additional custom chips, but can also be configured as a peripheral for an IBM PC. It can operate as a standalone system or as part of a network [12].

To enroll, a user is assigned a personal identification number and then puts a single finger on the glass or Plexiglas plate for scanning by a CCD image sensor. The $250-\mathrm{KB}$ image is digitalized and analyzed, and the result is approximately 1$\mathrm{KB}$ mathematical characterization of the fingerprint. This takes about 30 seconds. Identity verifications take less than 1 second. The equipment generally gives the user three attempts for acceptance or finds rejection. With the first attempt the false rejection is around 2-3 percent and false acceptance is less than 0.0001 per cent. Each standalone unit cab stores 48 fingerprint templates which may be expanded to 846 by installing an additional memory package [11].

Fingerprints have overcome the stigma of their use in law enforcement and military applications. Finger print recognition is appropriate for many applications and is familiar idea to most people even if only from crime dramas on television. It is non-intrusive, user friendly and relatively inexpensive.

\subsection{Benefits of Fingerprint}

Today fingerprint devices are by far the most popular form of biometric security in use, with a variety of systems on the market intended for general and mass-market usage.

Long gone are the huge bulky fingerprint scanners; now a fingerprint-scanning device can be small enough to be incorporated into a laptop for security.

2.4 Fact and Findings of Attendance System

Staff attendance software system is a software used to manage or monitor the time worked by employees for the purpose of efficiency and punctuality of the employees. These systems may be integrated with existing payroll processing software. Also, these systems track employee that come late to work and the ones that come early. These systems usually are able to give reports of all the staff records concerning signing in and out.

The advantages of staff attendance monitoring system are: it can reduce the time needed to enter 'hours worked' data into payroll system and can reduce errors in enforcement of company attendance policies[6].

Staff Attendance Monitoring System is an easy way to keep track of any organization's attendance records. It also works very well as a member database. The program was developed with three main goals in mind: versatility, ease of use, and security.

Versatility:-Not every organization has the same attendance policies, so this program easily allows you to specify your organization's policies. You can also store any information you would like about each member.

Ease of Use:-This program is very intuitive. It was designed so that all of the instructions that are needed are right on the screen. The finger print scanner which will be installed beside the computer is where employees will place their thumb in order to capture their details. 
Security:- A non-registered staff will not be allowed to sign in and out even though he place his/her thumb on the finger print scanner for years.

\section{MATERIALS AND METHOD}

Fingerprint identification system is mainly divided into three modules; fingerprint image preprocessing, minutiae extraction and minutiae matching [9]. The input fingerprint image is processed for skeleton image by the fingerprint image preprocessing stage and subsequently processed by minutiae extraction stage for extracting minutiae using crossing number concept. After minutiae extraction stage, if input fingerprint image is processed for enrollment then the skeleton image is saved as template fingerprint image in database, otherwise skeleton image is given to matching stage. In matching stage system compares skeleton image with template fingerprint images from database and make decision whether input fingerprint match or not.

Minutiae matching and detection is employed in this research which is the most common method of extraction using the Crossing Number $(\mathrm{CN})$ concept. This method involves the use of the skeleton image where the ridge flow pattern is eightconnected. The minutiae are extracted by scanning the local neighbourhood of each ridge pixel in the image using a $3 \times 3$ window. The $\mathrm{CN}$ value is then computed, which is defined as half the sum of the differences between pairs of adjacent pixels in the eight-neighbourhood [3]. The $\mathrm{CN}$ for a ridge pixel $\mathrm{P}$ is given by

$C N=\frac{1}{2} \sum_{i=1}^{8}|P i-1|, P_{9}=P_{1}$

where $p_{i}$ is the pixel value in the neighbourhood of $\mathrm{P}$. For a pixel $\mathrm{P}$, its eight neighbouring pixels are scanned in an anticlockwise direction as display in figure 1 :

After Cross Number for a ridge pixel has been computed, the pixel can then be classified according to the property of its $\mathrm{CN}$ value. Using the properties of the $\mathrm{CN}$ as shown in Table 1 , the ridge pixel can then be classified as a ridge ending, bifurcation or non-minutiae point. For example, a ridge pixel with a $\mathrm{CN}$ of one corresponds to a ridge ending, and a $\mathrm{CN}$ of three corresponds to a bifurcation.

Table 1: The table below shows the Properties of Crossing Number. [4].

\begin{tabular}{|l|l|}
\hline CN & Property \\
\hline 0 & Isolated Point \\
\hline 1 & Ridge Ending Point \\
\hline 2 & Continuing Ridge Point \\
\hline 3 & Bifurcation Point \\
\hline 4 & Crossing Point \\
\hline
\end{tabular}

\subsection{Minutiae Matching}

Let $\mathrm{T}$ and $\mathrm{I}$ be the representation of the template and input fingerprint, respectively. Each minutia is considered as a triplet $m=\{x, y, \theta\}$ that indicates the $\mathrm{x}$, $\mathrm{y}$ minutia location coordinates and the minutia angle $\theta$ :

$$
\begin{aligned}
& T=\{m 1, m 2, m 3 \ldots \ldots . . m m\}, m i=\{x 1, y 1, \theta 1\}, \\
& i=1,2 \ldots . . m \\
& I=\left\{m^{\prime} 1, m^{\prime} 2, m^{\prime} 3 \ldots \ldots . m^{\prime} n\right\}, m^{\prime} j=\left\{x^{\prime} j, y^{\prime} j, \theta^{\prime} j\right\}, \\
& j=1,2 \ldots . . n
\end{aligned}
$$

where $\mathrm{m}$ and $\mathrm{n}$ denote the number of minutiae in $\mathrm{T}$ and $\mathrm{I}$, respectively. A minutia $\mathrm{m}^{\prime} \mathrm{j}$ in $\mathrm{I}$ and a minutia $\mathrm{mi}$ in $\mathrm{T}$ are considered "matching", if the spatial distance (sd) between them is smaller than a given tolerance $\mathrm{r} 0$ and the direction difference (dd) between them is smaller than an angular tolerance $\theta 0$ [8].

\begin{tabular}{|l|l|l|}
\hline P4 & P3 & P2 \\
\hline P5 & P & P1 \\
\hline P6 & P7 & P8 \\
\hline
\end{tabular}

Figure 1: this figure displays 3 by 3 windows for minutiae searching

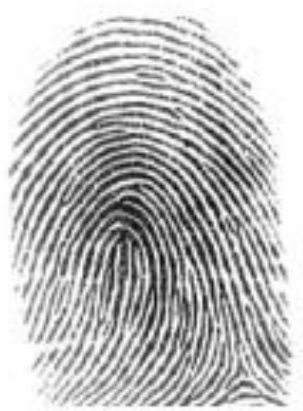

Figure 2: Original Image

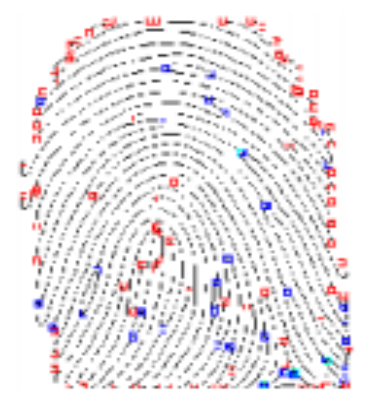

Figure 3: Minutiae Extracted using Cross Number concept 


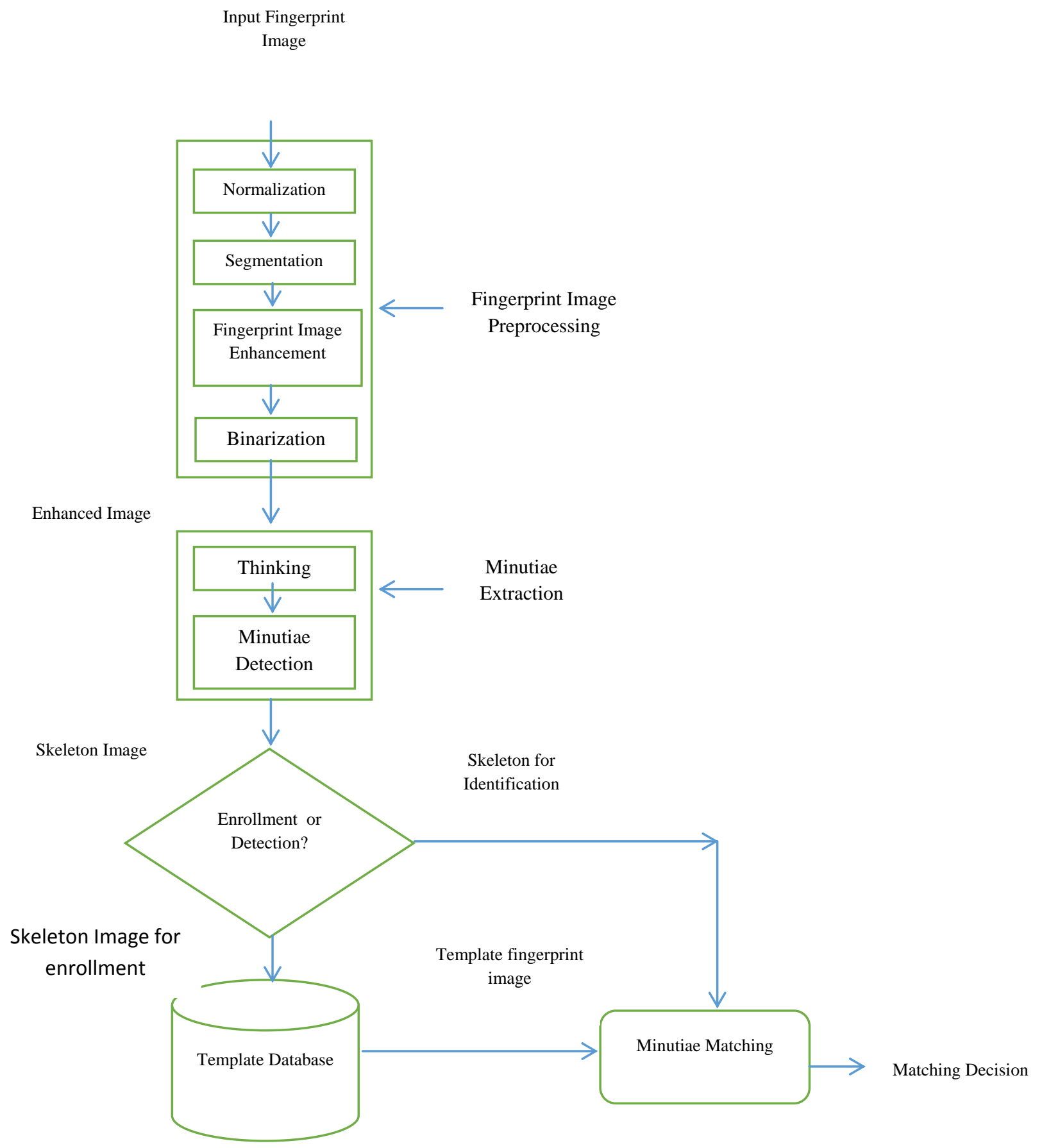

Figure 4: This figure display the Work Flow Diagram of Fingerprint Identification System. 


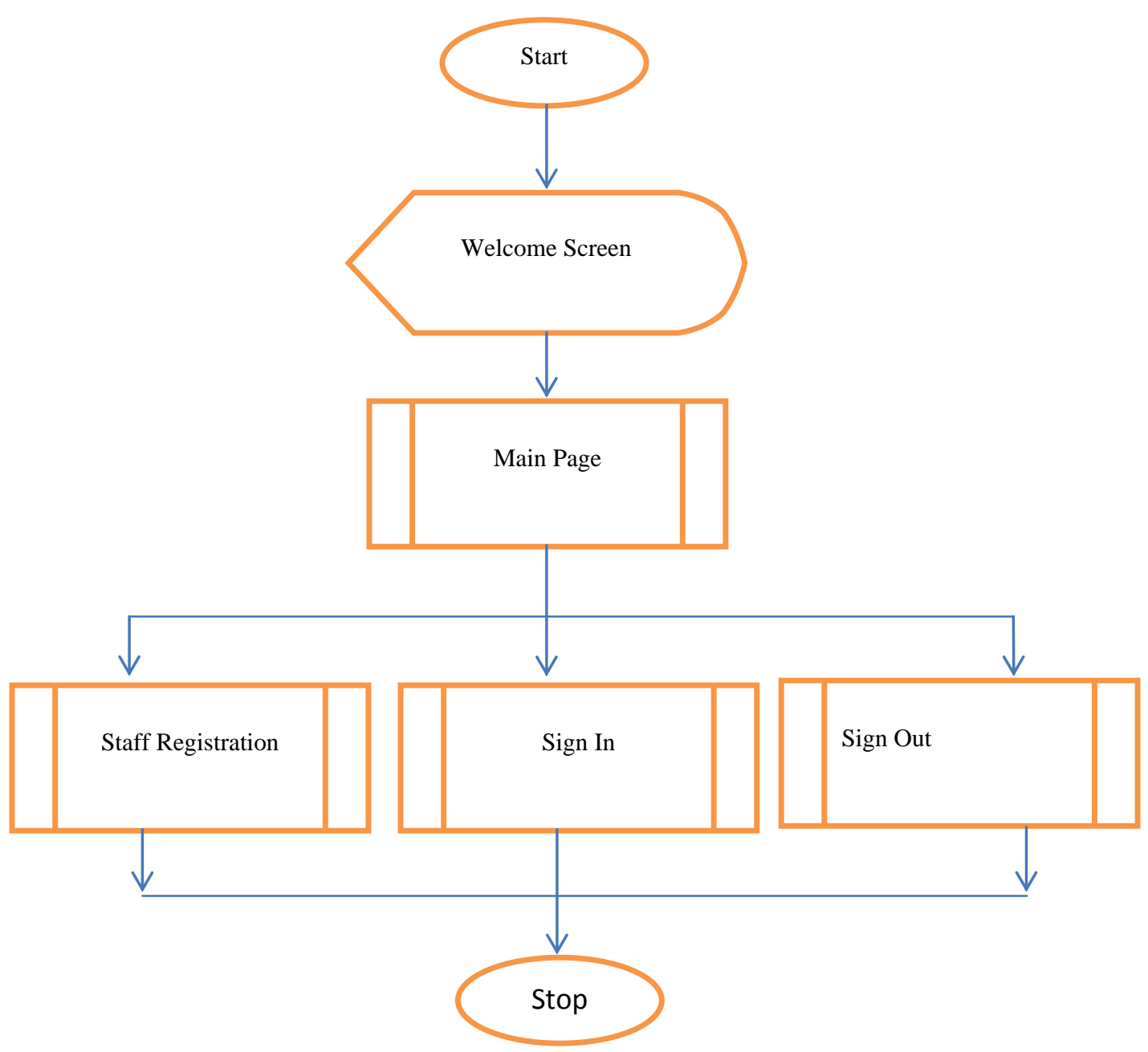

Figure 5: System flow chart of the proposed Fingerprint Attendance Monitoring System.

The flowchart comprises of three modules which are: The

Staff Registration Module for the Staff which is done by the System Administrator, The Sign In Module and the Sign Out Module mainly for the staff.

\section{DISCUSSION OF RESULT}

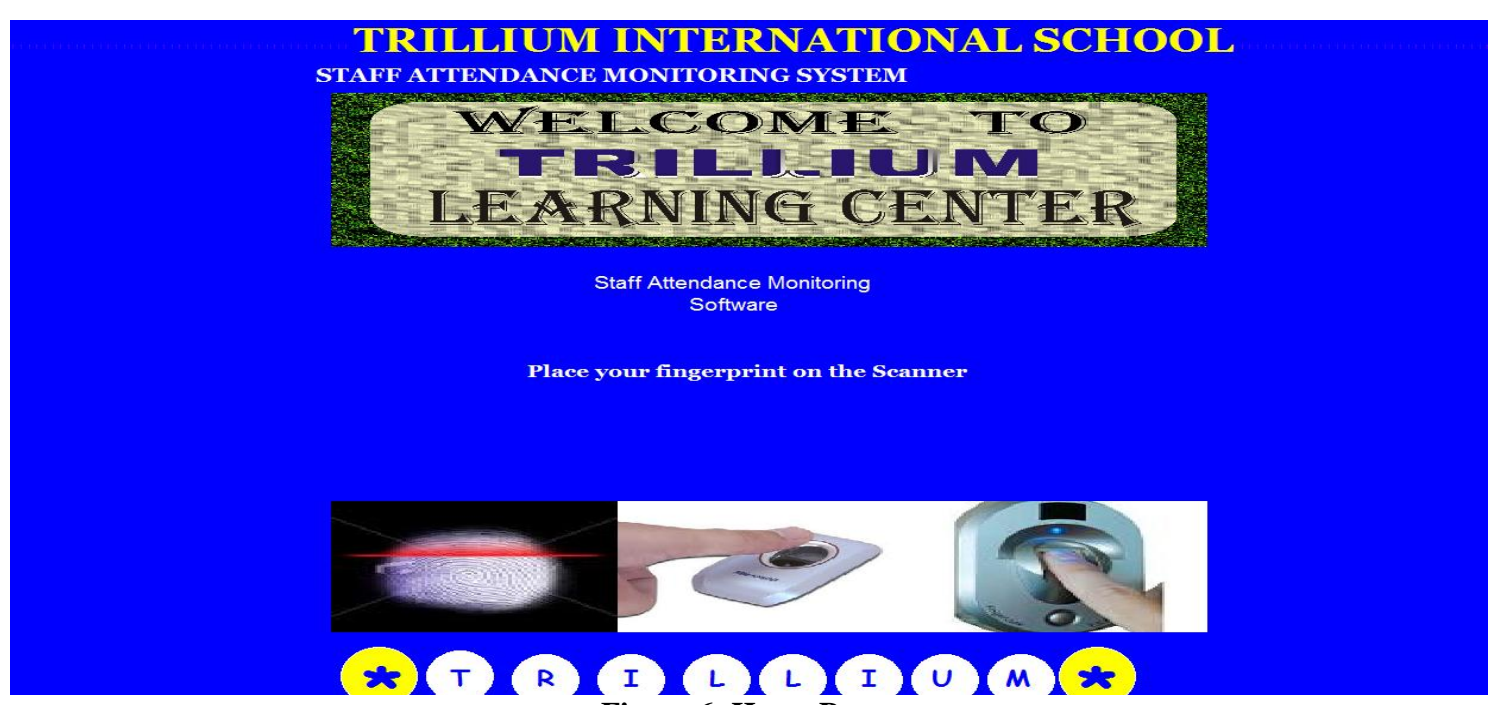

Figure 6: Home Page. 
Figure 6 display the homepage where Staff Place their Finger on the Scanning Machine. This module performs three activities, the Main Menu module, the sign In and as well the Sign Out. A staff will be asked to place his/her finger on the fingerprint scanner for sign in and as well place his/her finger on the fingerprint scanner for sign out on this main menu module.

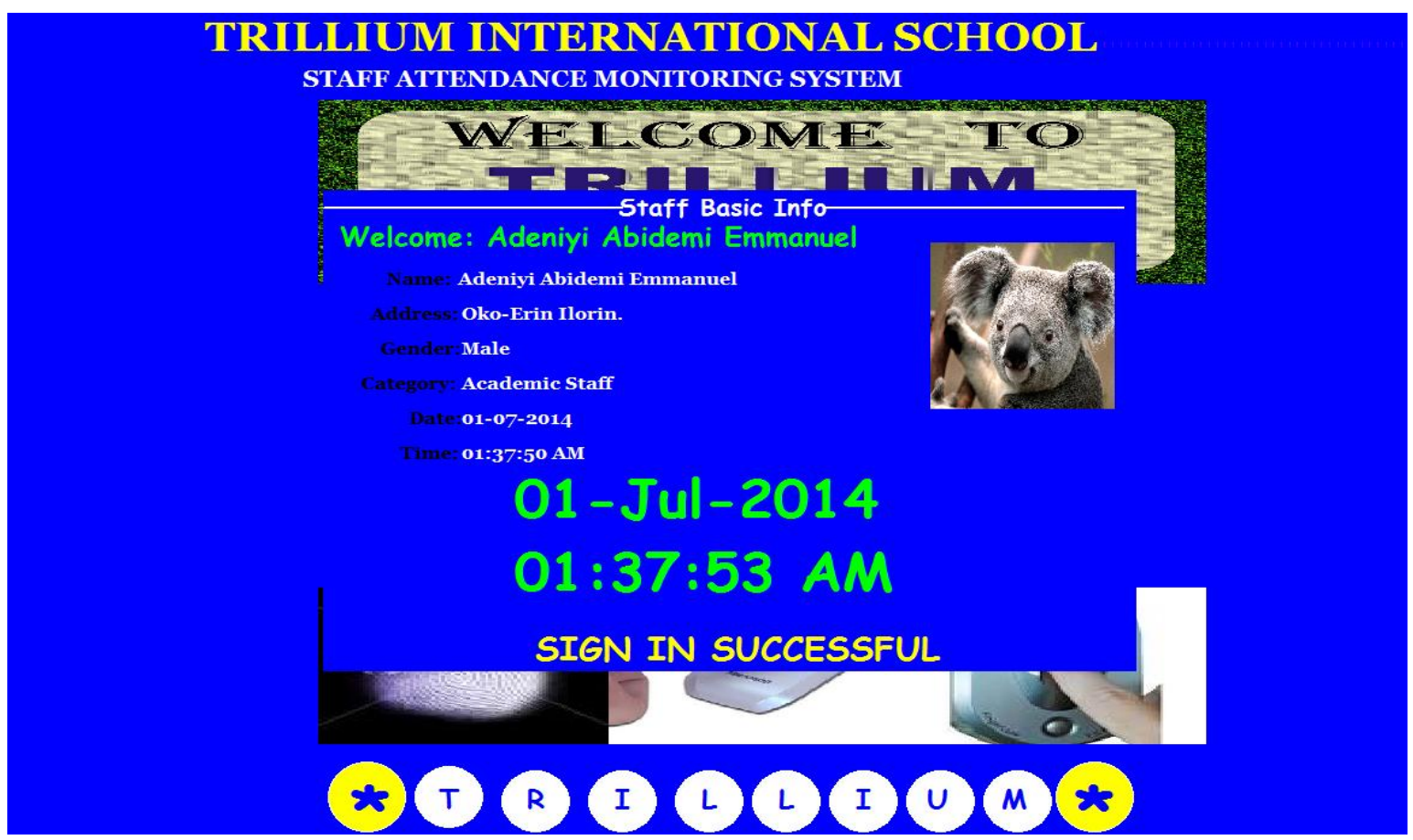

Figure 7: Staff Sign In Menu.

The popup interface is displayed after the staff might have place his/her finger on the fingerprint scanner is the Sign In
Interface with the staff basic information and as well the date and time the staff sign in as shown in figure 7.

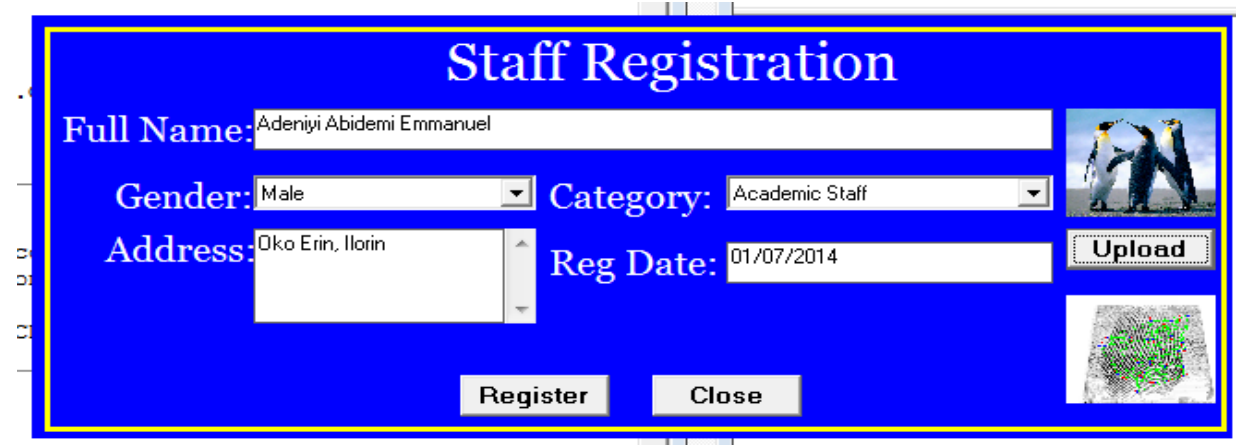

Figure 8: Staff Registration Module.

Figure 8 displays the form where the staff will be registered by the system Administrator. The basic information of the staff will be taken as well as the passport and the fingerprint before the registration can be successful. 


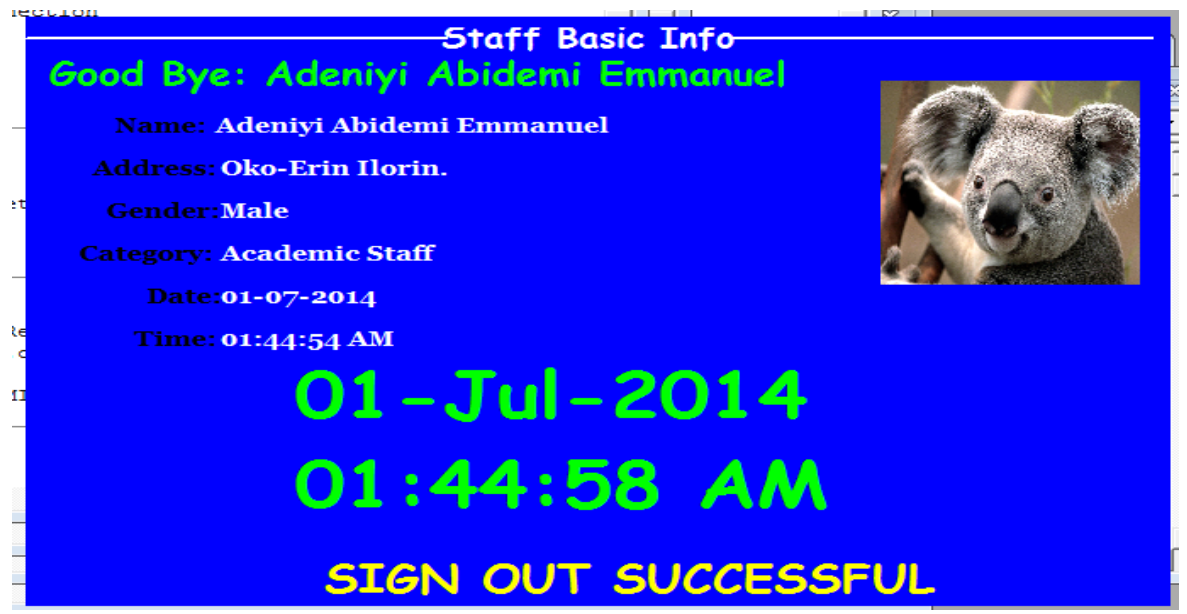

Figure 9: Staff Sign Out Module.

Figure 9 displays the basic information of a staff that has successfully signed out by placing his/her finger on the fingerprint scanner.

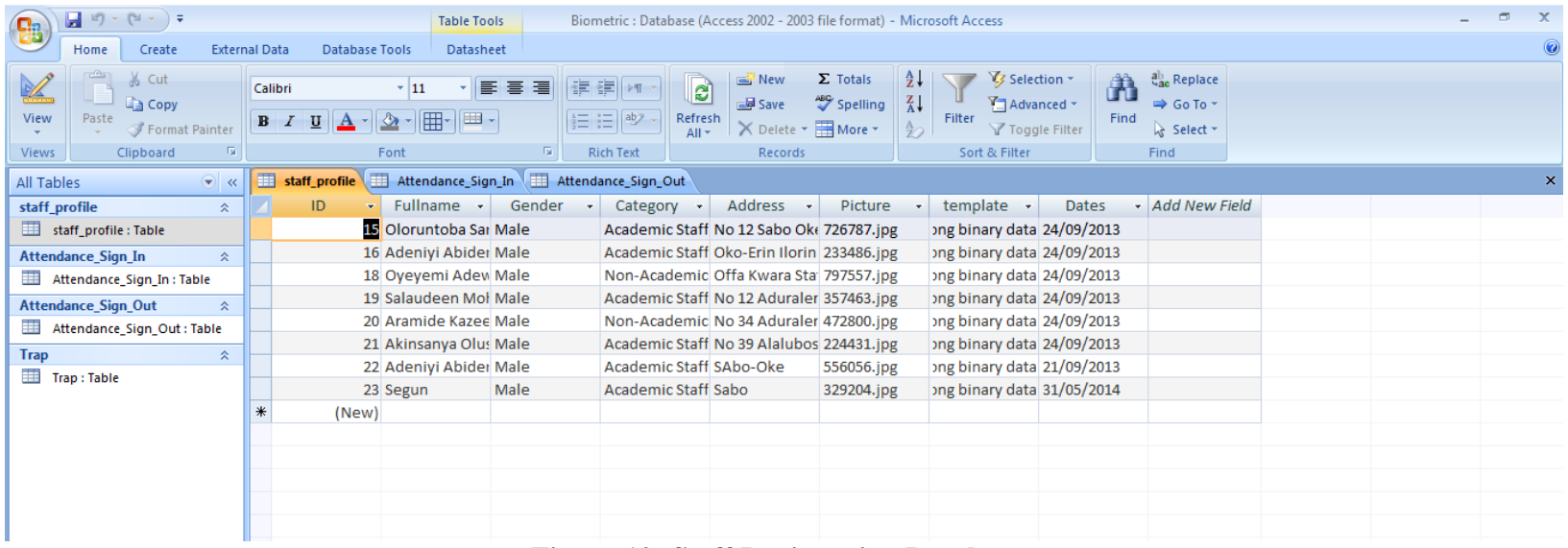

Figure 10: Staff Registration Database.

This figure displays the list of all registered staff, with their pictures displayed in jpg format and fingerprint template.

e.

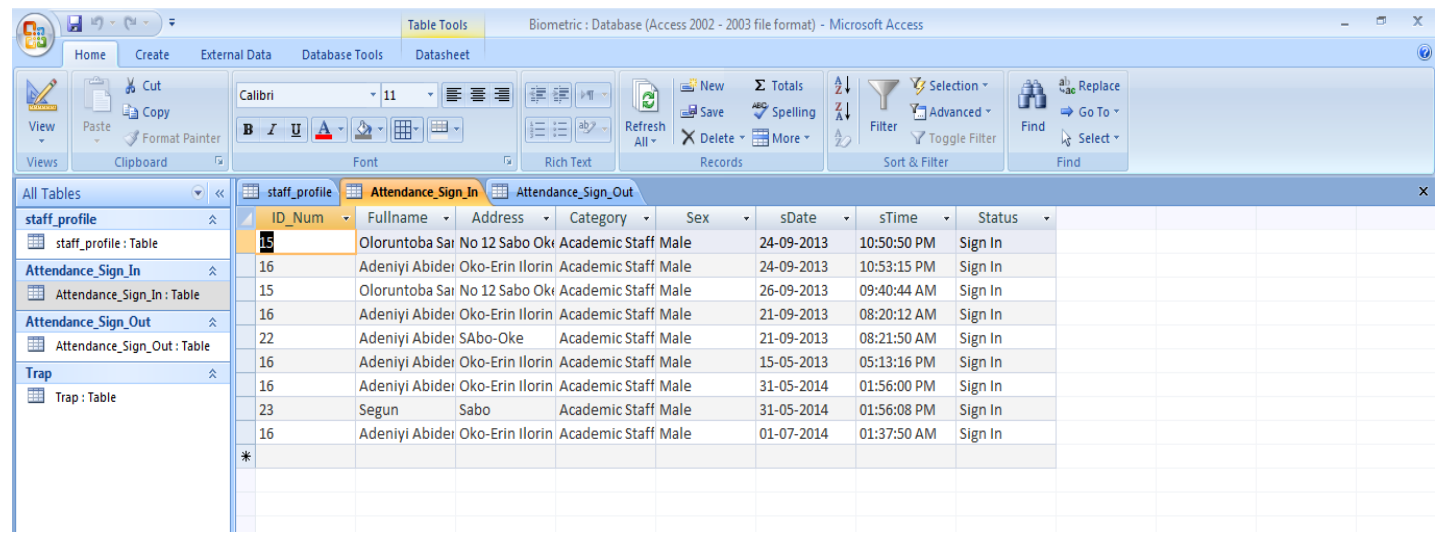

Figure 11: Staff Attendance Sign-In Database.

This figure displays the list of signed in staff on daily basis. The list comprises of the date and time each staff signed in. 


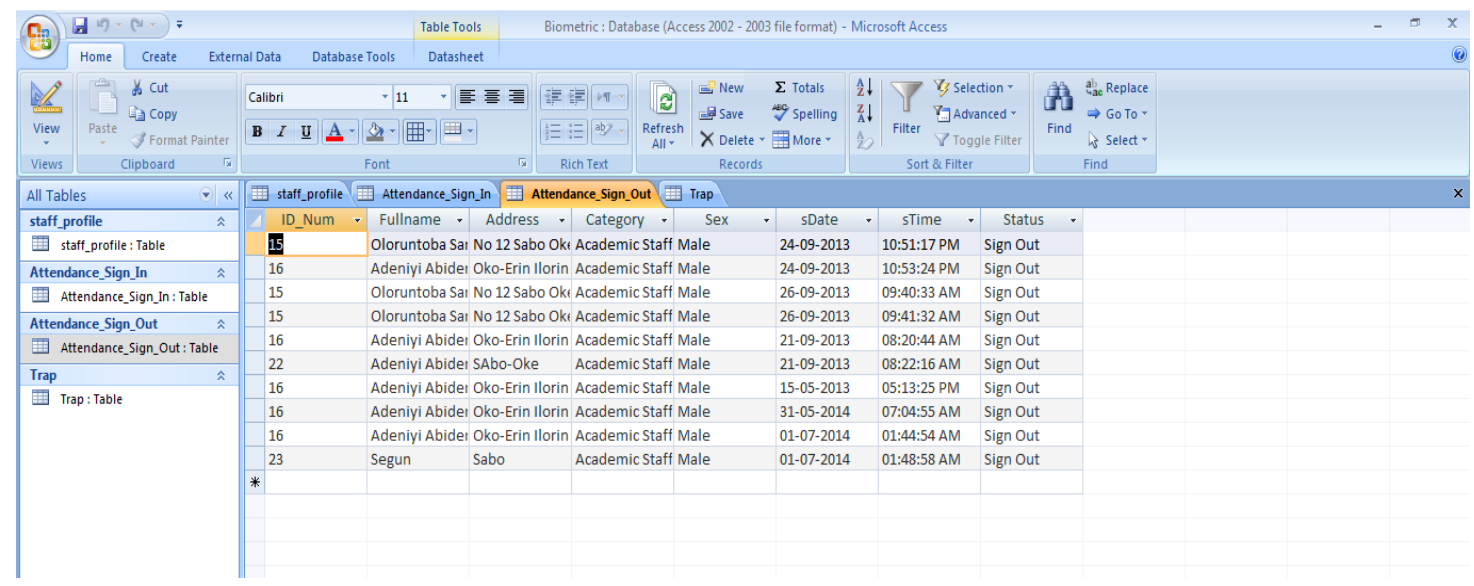

Figure 12: Staff Attendance Sign out Database.

This figure displays the list of staff that signed out from office on daily basis with the date and time each staff signed out from the office. And once a staff signed out for the day he/she cannot be able to sign-in for the day again.

\section{CONCLUSION AND RECOMMENDATION}

Having gone through the rudiments of the manual and automated biometrics based system; it can concluded that the need for a computer based attendance monitoring system cannot be over emphasized. From the two systems discussed, the manual system has a lot of draw backs and can be easily manipulated by the staff.

It is therefore concluded that the newly developed software is more effective and efficient in monitoring staff attendance. If it is implemented, the entire draw backs of the manual system will be eliminated or drastically minimized.

The future scope of this study can be implemented using facial recognition attendance system.

Due to the benefits of the automated system for monitoring staff attendance that was developed in this study, the system is hereby recommended to all private and government organizations in Nigeria in order to take proper records and manage the staff activities in their organization.

\section{ACKNOWLEDGMENTS}

Thanks to the experts who have contributed towards development of the template.

\section{REFERENCES}

[1] Aberdeen, F. (2008). Time Management System in Organization. Wesley Pub. London.

[2] Crowell, K. (2002). Importance of biometric Technology. Carnegie Mellon University.

[3] Feng Zhao and Xiaoou Tang, "Preprocessing and postprocessing for skeleton-based fingerprint minutiae extraction", Pattern Recognition Society, Published by Elsevier Ltd, 2006.

[4] Ishpreet Singh Virk and Raman Maini, "Fingerprint Image Enhancement and Minutiae Matching in Fingerprint
Verification", Journal of Computing Technologies, vol. 1, June 2012.

[5] James, M., Rehg and Takeo, K. (1993). DigitEyes: Vision-Based Human Hand-Tracking. School of Computer Science Technical Report CMU- CS-93-220, Carnegie Mellon University.

[6] Jonah, A. (1995). Fingerprint in Management attendance, Maxvin Pub. India.

[7] Jones, J. (1999). Statistical color models with application to skin detection. In Proceedings of IEEE Conference on Computer Vision and Pattern Recognition.

[8] Maltoni D., Maio D., Jain A. K., and Prabhakar S. "Handbook of Fingerprint Recognition". Springer, 2003.

[9] Manvjeet Kaur, Mukvinder Singh and Parvinder S. Sindhu, "Fingerprint Verification System using Minutiae Extraction Technique", Proceedings of World Academy of Science, Engineering and Technology, vol. 36, December 2008.

[10] Sato, Y. Kobayashi, Y. and Koike, H. (1991). Fast tracking of hands and fingertips in infrared images for augmented desk interface.

[11] Segen J. Segen, S. and Kumar, J. (1999). Shadow gestures: 3D hand pose estimation using a single camera.

[12] Sheetal, V. and Chander, K. (2012). Biometric Recognition system: An introduction. Department of computer science and applications k.U; Kurukshetra, Haryana, India.

[13] Swanirbhar, M. and Saurabh, P. (2016). Biometrics: Concepts, Methodologies, Tools and Application. Management Association, Information Resources. IGi Global Publisher. ppp 1852.

[14] Trucco E. (1998). Introductory Techniques for 3D Computer Vision. Prentice-Hall, Vision and Pattern Recognition.

[15] Zhang, Z. Shan, Y. and Shafer, S. (2001). Visual panel: Virtual mouse keyboard and 3rd controller with an ordinary piece of paper. In Proceedings of Perceptual User Interfaces. 